\title{
Impaction of Phytobezoar at Meckel's Diveticulum
}

\author{
Mohammad Masood Ur Rauf Hiraj Khan, Junaid Zia Hashmi, Naeem Lateef, Moazam Mumtaz and Farah Saleem
}

\begin{abstract}
Small bowel obstruction secondary to phytobezoars is a rare presentation in surgery. These are masses of undigested food and vegetables, which obstruct the narrowest part of gut. We discuss a case of a young patient presenting in emergency department with history of acute abdominal pain, distension and constipation. Diagnosis of acute intestinal obstruction was made on the basis of history, examination, and initial investigations. Exploratory laparotomy revealed a phytobezoar at the origin of Meckel's diverticulum, which was an incidental finding. He underwent wedge resection with primary closure and removal of phytobezoar. The change in dietary habits, decreased fiber intake, and psychiatric evaluation can prevent recurrence.
\end{abstract}

Key Words: Phytobezoar, Obstruction, Psychiatric evaluation.

\section{INTRODUCTION}

Among the intraluminal causes of intestinal obstruction, phytobezoars are rare. These account for only $2-4 \% .1$ Bezoars are firm masses which contain undigested fruits and vegetables. Factors which predispose to phytobezoars are high fiber intake, inadequate mastication, previous gastric surgery, hypochlorhydria, and loss of gastric pump mechanisms. ${ }^{2}$ According to one study, the previous gastric resection and surgeries such as partial gastrectomy or truncal vagotomy with pyloroplasty to treat gastric ulcers, increase the risk of bezoar formation. The incidence of bezoar formation after some sort of gastric surgery ranges between $5-12 \% .^{2}$

Primary bezoar develops due to stagnation of bile constituents and calcium salts in dilated bowel segments caused due to distal obstruction.

Herein, we report an intraoperative finding of phytobezoar impacted at the origin of Meckel's diverticulum in a 13year boy presenting with the features of intestinal obstruction.

\section{CASE REPORT}

A 13-year male, resident of Muzaffargarh, Punjab, presented in the Emergency Department of Nishtar Hospital, Multan, with the complaints of generalised abdominal pain and vomiting for 3 days, and constipation and abdominal distention for 2 days. There was no history of fever, weight loss, or any previous abdominal surgery. There was no previous history of pulmonary tuberculosis. Patient was non-smoker, non-addict, and

Department of General Surgery, Nishtar Hospital, Multan, Pakistan

Correspondence: Dr. Junaid Zia Hashmi, Department of

General Surgery, Nishtar Hospital, Multan, Pakistan

E-mail: juneehashmi@yahoo.com

Received: May 31, 2018; Accepted: September 18, 2018 no significant history about the eating habits of the patient was given. Systemic history was non-significant.

The clinical examination showed a young boy of average built and height with stable vital signs. The abdomen was distended with central inverted umbilicus. Abdomen was tense, and mildly tender. Hernial orifices were intact and no lymph nodes were palpable. Bowel sounds were absent. Perrectal examination revealed empty rectum with no palpable mass. Oral, central nervous system, respiratory and cardiovascular examinations were unremarkable.

His hemogalobin was $12.7 \mathrm{~g} / \mathrm{dl}$ and total leukocyte count (TLC) was $8800 \mathrm{~mm}^{3}$. Renal and liver function tests were normal. Abdominal erect $\mathrm{X}$-ray showed dilated jejunal and ileal gut loops. Chest X-ray was unremarkable. Ultrasound abdomen revealed dilated gut loops with absent peristalsis and minimal interloop-free fluid.

Exploratory laparotomy confirmed the finding of $20 \times 3 \mathrm{~cm}$ phytobezoar in small intestine, two feet proximal to ileocaecal junction and incidental finding of Meckel's diverticulum. Wedge resection was done, phytobezoar removed through it, followed by primary repair (Figures 1 and 2). Only complaint in postoperative period was pain, which was dealt with analgesic. On fourth postoperative day he was allowed oral sips; and soft diet was allowed on fifth postoperative day. He was discharged six days later and was found healthy and well during follow-up in surgical outdoor clinic after one week.

\section{DISCUSSION}

Small bowel obstruction is a common condition encountered in surgical practice. Four types of bezoars are usually encountered in surgical practice. Phytobezoars are the most common type of bezoars. These are composed of non-digestible vegetable matter such as cellulose, hemicellulose, lignin and tannin, which polymerise in the presence of gastric acid and cause obstruction. ${ }^{3}$ Trichobezoars are concretion of hair fibres in the 


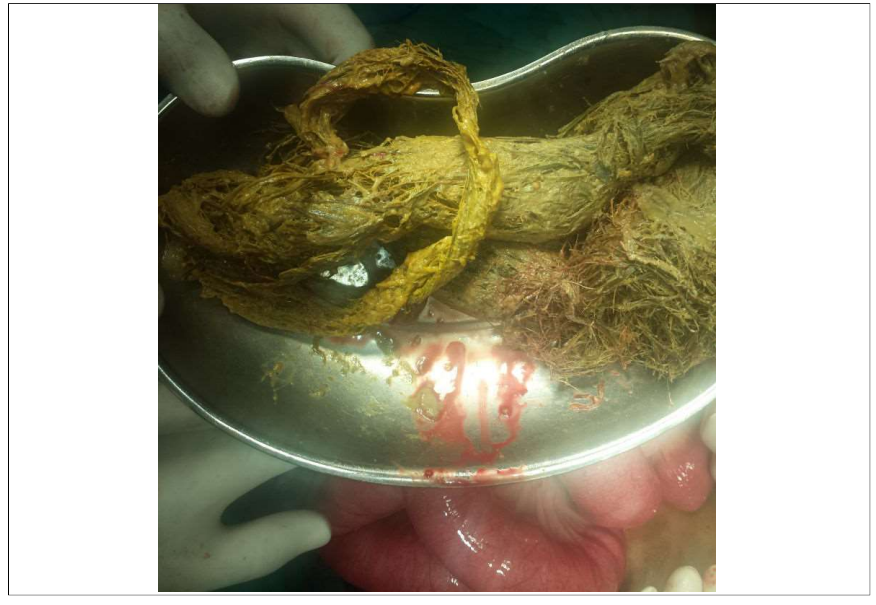

Figure 1: Phytobezoar removed from the small intestine.

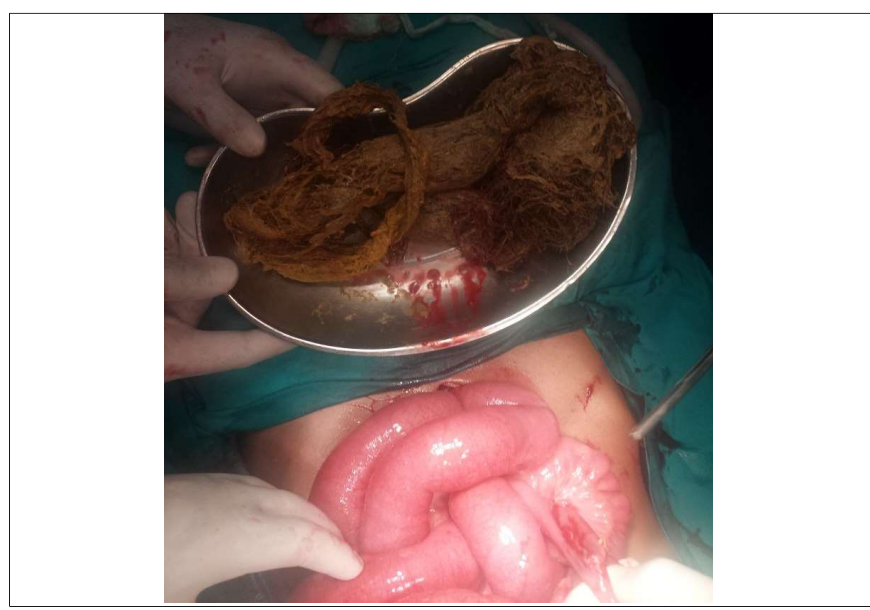

Figure 2: Close-up view of the phytobezoar

stomach which usually present in patients with history of psychiatric condition called trichotillomania. ${ }^{4}$ Pharmacobezoars are the medication bezoars. Antacids lead to pharmacobezoar formation. Lactobezoars are milk curd caused by infant formula. Bezoars impact in the narrowest part of the intestine. Terminal ileum and ileocecal valve being the narrowest parts of intestine often serves as the site of obstruction due to impaction of these bezoars. The stricture caused by Meckel's diverticulum was most probably the narrowest part in this patient. It is interesting to note that most reported cases of phytobezoars have a history of gastric surgery. The patient did not give previous history of any gastoric surgery.

In this patient, phytobezoar which was causing small bowel obstruction was found at $60 \mathrm{~cm}$ from the ileocecal valve. The origin of Meckel's diverticulum, which was an incidental finding, served as the narrowest part in this patient. According to a study, distal ileum approximately $50-70 \mathrm{~cm}$ from the ileocecal valve, serves as the most common site of impaction of bezoars due to the small diameter, reduced peristalsis and increased water absorption. ${ }^{5}$

There is wide variety of treatment options for phytobezoar management. It includes conservative, medical, and surgical approaches. Conservative approach is usually not widely accepted. It is reasonable only in the case if the presenting symptoms resolve before the patient has underwent some definitive treatment. Laparoscopy is an effective modality in the management of phytobezoar. 6 An alternative approach is laparotomy followed by enterotomy with primary closure. This approach is also useful in making definitive diagnosis and treatment. ${ }^{7}$ This patient underwent laparotomy, wedge resection of Meckel's diverticulum, removal of phytobezoar, and primary repair. Simple preventive measures can decrease the risk of bezoar formation. High fibre diet is a risk factor in the development of bezoars and should be avoided by patients who have undergone any kind of gastric surgery. Increased uptake of water decreases the risk of bezoar formation. Adequate mastication of food, treatment of underlying gastrointestinal motility disorders and psychiatric evaluation is necessary to avoid bezoar formation.

Small bowel phytobezoar is an unusual cause of acute intestinal obstruction. Diagnosis, based on detailed history, physical examination, plain abdominal X-ray, $X$-ray abdomen erect, and ultrasound findings, is of nonspecific small bowel obstruction. Abdominal scan can sometimes be helpful in making diagnosis. Laparotomy is required in resolving the diagnostic confusion: and it is also needed for the definitive treatment.

\section{REFERENCES}

1. Ben-Porat T, Sherf Dagan S, Goldenshluger A, Yuval JB, Elazary R. Gastrointestinal phytobezoar following bariatric surgery: Systematic review. Surg Obes Relat Dis 2016 12: 1747-54.

2. Hill J. Intestinal Obstruction. In: Williams N.S, Bulstrode C.J.K, O'Connel P.R, Eds. Bailey and Love's Short Practice of Surgery, Ed. 26th. India: CRC Press Taylor \& Francis Group; 2013: pp.1183.

3. Ladas SD, Kamberoglou D, Karamanolis G, Vlachogiannakos J, Zouboulis-Vafiadis I. Systematic review: Coca-cola can effectively dissolve gastric phytobezoars as a first-line treatment. Aliment Pharmacol Ther 2013; 37:169-73.

4. Sah DE, Koo J, Price VH. Trichotillomania. Dermatol Ther 2008; 21:13-21.

5. Das SS, Husain M, Bhat A, Hajini FF. Phytobezoar causing terminal lleal obstruction: Can enterotomy be avoided? JSM Clin Case Rep 2014; 2:1024.

6. Sheikh AB, Akhtar A, Nasrullah A, Haq S, Ghazanfar H. Role of laparoscopy in the management of acute surgical abdomen secondary to phytobezoars. Cureus 2017; 9:e1363.

7. Erzurumlu K, Malazgirt Z, Bektas A, Dervisoglu A, Polat C, Senyurek G, et al. Gastrointestinal bezoars: A retrospective analysis of 34 cases. World J Gastroenterol 2005; 11:1813-7.

.......... 